\section{Independent association of glucocorticoids with damage accrual in SLE}

To cite: Apostolopoulos D, Kandane-Rathnayake R, Raghunath $\mathrm{S}$, et al. Independent association of glucocorticoids with damage accrual in SLE. Lupus Science \& Medicine 2016;3: e000157. doi:10.1136/lupus2016-000157

- Additional material is available. To view please visit the journal (http://dx.doi.org/ 10.1136/lupus-2016000157).

Received 18 March 2016 Revised 13 October 2016 Accepted 26 October 2016

\section{(a) CrossMark}

${ }^{1}$ School of Clinical Sciences at Monash Health, Monash University Faculty of Medicine, Nursing and Health Sciences, Melbourne, Australia

${ }^{2}$ Department of

Rheumatology, Monash Health, Melbourne, Australia ${ }^{3}$ Department of Medicine and Rheumatology, The University of Melbourne at St Vincent's Hospital, Melbourne, Australia

Correspondence to Dr Diane Apostolopoulos; Diane.apostolopoulos@ monash.edu

\section{ABSTRACT}

Objectives: To determine factors associated with damage accrual in a prospective cohort of patients with SLE.

Methods: Patients with SLE who attended the Lupus Clinic at Monash Health, Australia, between 2007 and 2013 were studied. Clinical variables included disease activity (Systemic Lupus Erythematosus Disease Activity Index-2K, SLEDAI-2K), time-adjusted mean SLEDAI, cumulative glucocorticoid dose and organ damage (Systemic Lupus International Collaborating Clinics Damage Index (SDI)). Multivariate logistic regression analyses were performed to identify factors associated with damage accrual.

Results: A total of 162 patients were observed over a median (IQR) 3.6 (2.0-4.7) years. Seventy-five per cent $(n=121)$ of patients received glucocorticoids. Damage accrual was significantly more frequent in glucocorticoid-exposed patients ( $42 \%$ vs $15 \%$, $\mathrm{p}<0.01$ ). Higher glucocorticoid exposure was independently associated with overall damage accrual after controlling for factors including ethnicity and disease activity and was significant at time-adjusted mean doses above $4.42 \mathrm{mg}$ prednisolone/day; the $\mathrm{OR}$ of damage accrual in patients in the highest quartile of cumulative glucocorticoid exposure was over 10 . Glucocorticoid exposure was independently associated with damage accrual in glucocorticoid-related and nonglucocorticoid related domains of the SDI.

Conclusions: Glucocorticoid use is independently associated with the accrual of damage in SLE, including in non-glucocorticoid related domains.

\section{INTRODUCTION}

SLE is a chronic multisystem autoimmune disease in which tissue inflammation results in organ dysfunction. Permanent organ damage occurs in $61 \%$ of patients within 7 years of the diagnosis of SLE ${ }^{1}$ and it is well established that the accrual of damage, measured using instruments such as the Systemic Lupus International Collaborating Clinics/ American College of Rheumatology Damage Index (SDI), ${ }^{2}$ contributes to mortality in

\section{SIGNIFICANCE AND INNOVATION}

Glucocorticoid use in lupus is associated with the accrual of irreversible organ damage, independent of disease activity. Glucocorticoid use is associated with harm in both domains of the SDI traditionally associated with glucocorticoid-induced harm (cataracts, osteoporotic fracture, avascular necrosis, diabetes mellitus) and the residual SDI domains not previously associated with glucocorticoid-induced harm.

- The development of irreversible organ damage associated with glucocorticoid therapy occurs in a dose-dependent manner, with higher cumulative exposure associated with greater damage.

- Even lower doses of glucocorticoid than previously suspected are associated with damage accrual in SLE. The threshold identified was a time-adjusted mean prednisolone of $4.4 \mathrm{mg} /$ day.

SLE. ${ }^{34}$ In the SDI, items scored do not have to be attributable to lupus disease activity in order to contribute to the aggregate measure; thus, treatment-related outcomes may also contribute. Damage accrual has been clearly shown to be associated with the cumulative effects of disease activity, ${ }^{4-6}$ but recent studies have also suggested that damage may be associated with SLE treatment. ${ }^{7}{ }^{8}$ Indeed, some items scored in the SDI, such as osteoporosis, are more closely associated with glucocorticoid exposure than with active disease.

Glucocorticoid therapy remains a mainstay of SLE treatment in both the acute and chronic setting, and thus the majority of patients receive these medications for significant periods. It is well known that glucocorticoid use results in numerous predictable adverse effects, ${ }^{8}{ }^{9}$ and hence glucocorticoid use may contribute significantly to the morbidity of SLE. Indeed, recent studies have found associations between glucocorticoid therapy and permanent organ damage in lupus, ${ }^{8}$ with some evidence that these 
effects are dose-dependent. ${ }^{10}$ However, it has been difficult to directly attribute organ damage to glucocorticoid use in SLE, as treatment with glucocorticoids generally occurs in the context of high disease activity.

The potentially independent contribution of glucocorticoid therapy to SLE organ damage has not been fully resolved. The objective of the present study was to quantify damage accrual in a prospectively followed cohort of patients with SLE and determine the association of glucocorticoid use with damage.

\section{METHODS}

Study setting

At the Monash Lupus Clinic in Melbourne, Australia, all patients are invited to enrol in the Monash Lupus cohort study. As previously described, ${ }^{11}{ }^{12}$ upon written informed consent, data (clinical and laboratory) are collected according to a standardised protocol at each review, undertaken at 2-6 monthly intervals as determined by the physician. Consenting patients (age $>18$ years) that met American College of Rheumatology (ACR) criteria for SLE (ACR criteria $\geq 4)^{13}$ enrolled in the Monash Lupus database from January 2007 until November 2013 were eligible for this study. The study period was defined as the time between the first and last recorded SDI measurement for each individual patient. The project was approved by the Monash Health Human Research Ethics Committee.

There were a total of 222 patients enrolled in the study period. Thirty were excluded as they either failed to meet ACR criteria or to provide consent, and 30 patients were excluded because they had been reviewed on only a single occasion. The final number of patients included was 162 .

\section{Data collection}

Baseline demographic data: The data included age, gender, self-reported ethnicity, ${ }^{11}$ date of onset of SLE, date of SLE diagnosis, number of ACR criteria and smoking status.

Disease activity: At each review, disease activity was assessed using the Systemic Lupus Erythematosus Disease Activity Index-2K (SLEDAI-2K). ${ }^{14}$ Where individual components of the SLEDAI-2K were incomplete, the previous value was carried forward, and where the firstvisit SLEDAI-2K was incomplete, those visits were not included. A time-adjusted mean SLEDAI (AMS) ${ }^{15}$ was calculated as a measure of disease activity over time. Active disease was defined as AMS $>4$, as defined by Yee et $a .^{16}{ }^{\mathrm{A}}$ three-point physician global assessment (PGA) of disease activity as used in the Safety of Estrogens in Lupus Erythematosus National Assessment (SELENA)SLEDAI $^{17}$ was also completed at each visit, and a timeadjusted mean PGA was calculated as a measure of PGA over time.

Damage accrual: $\mathrm{The}_{\mathrm{SDI}}{ }^{2}$ which comprises 38 items in 12 domains was used to measure organ damage. SDI was scored at baseline and on an annual basis thereafter.
Baseline and last-visit SDI data allowed for analysis of damage accrual, with change in SDI $>0$ chosen as defining damage accrual on the basis of this being previously demonstrated to be clinically significant. ${ }^{3}$ Four domains of the SDI focus specifically on glucocorticoid-related adverse effects, namely, osteoporotic fracture, avascular necrosis, diabetes mellitus and cataracts. From the sum of these domains, we generated a glucocorticoid-related SDI (GC-SDI) as recently described; ${ }^{7}$ we also analysed damage accrual in the residual domains of SDI after subtraction of GC-SDI, referred to here as non-GC-SDI.

Treatment data: At each review, data were collected on all medications and doses. Information regarding in-hospital dosage of intravenous methylprednisolone was obtained through review of all patient medical records, and exposure was converted to prednisolone-equivalent dose for analysis. Patients with any glucocorticoid use were compared with patients with no glucocorticoid exposure, and cumulative glucocorticoid use was grouped by quartiles to allow for categorical analysis. Timeadjusted mean prednisolone was calculated as a measure of prednisolone exposure over time.

\section{Statistical analysis}

All statistical analyses were performed using Stata V.13.1 (StataCorp, College Station, Texas, USA). Continuous variables were described as median (IQR, range) and compared using Wilcoxon rank-sum tests. Categorical variables were described as frequency (\%) and compared using $\chi^{2}$ tests. In addition, $\chi^{2}$ test for trend was also performed to assess the overall difference in damage accrual among the GC quartiles. Univariable and multivariable logistic regression analyses were carried out to identify factors associated with damage accrual. Variables with $\mathrm{p}<0.1$ in univariate logistic regression analyses were included in multivariate analysis. Results were reported as ORs with 95\% CIs. Values of $\mathrm{p} \leq 0.05$ were regarded as statistically significant. Correlation between each of AMS and time-adjusted mean PGA with cumulative prednisolone dose was tested using the Pearson correlation coefficient, and found to be 0.52 and $0.49 \quad(p<0.01)$, respectively (see online supplementary figure S1). As AMS and time-adjusted mean PGA were highly correlated $(\mathrm{r}=0.76, \mathrm{p}<0.01)$ (see online supplementary figure $\mathrm{S} 1$ ), the decision was made to only include AMS in the multivariate analysis. To evaluate our multivariable logistic regression model's prediction performance, the Hosmer-Lemeshow $\chi^{2}$ test was performed which indicated that our model adequately fit the data $(\mathrm{p}=0.5)$. In addition, a receiveroperating characteristic (ROC) test indicated high predictive accuracy $(\mathrm{ROC}=0.81)$.

\section{RESULTS}

\section{Patient characteristics}

In total, 162 patients were included in this study, and their baseline characteristics are presented in table 1. 
Table 1 Patient characteristics, stratified by glucocorticoids exposure

\begin{tabular}{|c|c|c|c|c|}
\hline & \multirow[b]{2}{*}{$\begin{array}{l}\text { Total (N=162) } \\
\text { Median (IQR) (range) }\end{array}$} & \multicolumn{2}{|c|}{ Exposed to glucocorticoids ever? } & \multirow[b]{2}{*}{ p Value } \\
\hline & & $\begin{array}{l}\text { No }(\mathrm{N}=41) \\
\text { Median (IQR) (range) } \\
\end{array}$ & $\begin{array}{l}\text { Yes (N=121) } \\
\text { Median (IQR) (range) } \\
\end{array}$ & \\
\hline Age at diagnosis (years) & $35(27,47)(10,69)$ & $38(30,52)(14,69)$ & $33(27,44)(10,69)$ & 0.09 \\
\hline Age at recruitment (years) & $43(34,54)(23,78)$ & $47(36,55)(23,78)$ & $42(33,54)(23,77)$ & 0.13 \\
\hline $\begin{array}{l}\text { Duration of observation } \\
\text { (years) }\end{array}$ & $3.6(2.0,4.7)(0.4,6.3)$ & $3.5(1.7,4.5)(1.0,5.7)$ & $3.9(2.1,5.0)(0.04,6.3)$ & 0.13 \\
\hline Number of clinic visits & $14(8,22)(2,47)$ & $10(5,13)(2,23)$ & $16(10,25)(2,47)$ & $<0.01$ \\
\hline Cumulative prednisolone (g) & $4.8(0,9.2)(0,56.6)$ & 0 & $7.3(3.6,11.9)(0.17,56.6)$ & $<0.01$ \\
\hline $\begin{array}{l}\text { Time-adjusted mean } \\
\text { prednisolone (mg/day) }\end{array}$ & $4.4(0,7.8)(0,40)$ & 0 & $5.7(3.6,9.4)(0.04,40)$ & $<0.01$ \\
\hline Number of ACR criteria & $5(4,6)(4,9)$ & $5(4,5)(4,8)$ & $5(4,6)(4,9)$ & 0.16 \\
\hline Baseline SLEDAI & $4(2,8)(0,22)$ & $2(0,4)(0,16)$ & $4(2,8)(0,22)$ & $<0.01$ \\
\hline $\begin{array}{l}\text { Time-adjusted mean SLEDAI } \\
\text { (AMS) }\end{array}$ & $3.5(1.8,5.3)(0,13.9)$ & $1.6(0.8,3.1)(0,6.0)$ & $4.3(2.4,5.8)(0,13.9)$ & $<0.01$ \\
\hline Time-adjusted mean PGA & $0.43(0.27,0.68)(0,1.87)$ & $0.25(0.11,0.42)(0,0.84)$ & $0.50(0.34,0.73)(0,1.87)$ & $<0.01$ \\
\hline Baseline SDI & $1(0,1)(0,4)$ & $0(0,1)(0,3)$ & $1(0,1)(0,4)$ & 0.4 \\
\hline \multirow[t]{2}{*}{ Final SDI } & $1(0,2)(0,12)$ & $0(0,1)(0,4)$ & $1(0,2)(0,12)$ & 0.03 \\
\hline & $\mathbf{N}(\%)$ & $\mathbf{N}(\%)$ & $\mathbf{N}(\%)$ & \\
\hline Female & $138(85 \%)$ & $35(85 \%)$ & $103(85 \%)$ & 0.9 \\
\hline Ethnicity & & & & 0.05 \\
\hline Caucasian & $89(55 \%)$ & $29(71 \%)$ & $60(50 \%)$ & \\
\hline Asian & $65(40 \%)$ & $10(24 \%)$ & $55(45 \%)$ & \\
\hline Other & $8(5 \%)$ & $2(5 \%)$ & $6(5 \%)$ & \\
\hline Current smoker* & $26(21 \%)$ & $6(21 \%)$ & $20(21 \%)$ & 0.9 \\
\hline Hydroxychloroquine ever & $153(94 \%)$ & $38(93 \%)$ & $115(95 \%)$ & 0.6 \\
\hline $\begin{array}{l}\text { Other immunosuppressants } \\
\text { ever }\end{array}$ & $121(75 \%)$ & $13(32 \%)$ & $108(89 \%)$ & $<0.01$ \\
\hline No. of patients with AMS >4 & $70(43 \%)$ & $4(10 \%)$ & $66(55 \%)$ & $<0.01$ \\
\hline No. of patients with $\Delta S D I>0$ & $57(35 \%)$ & $6(15 \%)$ & $51(42 \%)$ & $<0.01$ \\
\hline $\begin{array}{l}\text { No. of patients with } \\
\Delta \mathrm{GC}-\mathrm{SDI}>0\end{array}$ & $14(9 \%)$ & $2(5 \%)$ & $12(10 \%)$ & 0.3 \\
\hline \multicolumn{5}{|c|}{$\begin{array}{l}\text { *Data missing for } 27 \text { patients. } \\
\text { p Values were calculated using } \chi^{2} \text { tests for the difference in proportions and Wilcoxon rank-sum tests for the difference in medians between } \\
\text { groups. } \\
\Delta \text {, change; ACR, American College of Rheumatology; GC-SDI, glucocorticoid-related SDI; PGA, physician global assessment; SLEDAI, } \\
\text { Systemic Lupus Erythematosus Disease Activity Index. }\end{array}$} \\
\hline
\end{tabular}

Eighty-five per cent of patients were female, with a median (IQR) age at enrolment of 43 years $(34,54)$. Ethnicity was diverse, with patients of Asian descent accounting for $40 \%$ of the study population. Antimalarial use was frequent, with $94 \%$ exposed to hydroxychloroquine during the period of observation. Also, methotrexate, azathioprine, mycophenolate, cyclophosphamide and rituximab use was common, with $26 \%, 41 \%, 25 \%, 6 \%$ and $4 \%$ respectively exposed during the period of observation.

At baseline, $40 \%$ of patients had active disease, defined as SLEDAI-2K $>4$, while $51 \%$ of patients had baseline SDI $>0$ (median (IQR) (range) baseline SDI=0 $((0,1)(0,4))$ (table 1$)$.

One hundred and twenty-one patients (75\%) were exposed to glucocorticoids during the period of observation. Baseline disease activity was higher in glucocorticoid-exposed patients when compared with those never exposed to glucocorticoids $(\mathrm{p}<0.01)$ (table 1$)$. Over the study period, AMS was significantly higher in glucocorticoid-exposed patients ( 4.3 vs $1.6, \mathrm{p}<0.01$ ), as was the frequency of immunosuppressant use $(p=0.01)$. Concordant with this, the number of clinic visits over the period observed was significantly higher in glucocorticoid-exposed patients (16 vs $10, \mathrm{p}<0.01$ ).

\section{Factors associated with damage accrual}

Damage accrual was observed in 57 patients. Damage accrual was significantly different between glucocorticoid-exposed and non-exposed patients (table 1). Damage accrual was observed in $42 \%$ of glucocorticoid-exposed patients, compared with $15 \%$ of patients not exposed to glucocorticoids $(p<0.01)$. In addition, $\chi^{2}$ test for trend showed a statistically significant difference $(p<0.01)$ in damage accrual among GC dose categories (quartiles). Although the proportion of patients with change in GC-SDI was higher in glucocorticoid-exposed patients $(12 / 121$ vs $2 / 41)$, this was not statistically significant (table 1). Damage accrual was also significantly more frequent among patients with damage at baseline (baseline 
SDI $>0)$. Of 82 patients $(51 \%)$ with $\mathrm{SDI}>0$ at enrolment, $39(48 \%)$ accrued further damage during the study period. In contrast, only 18/80 (22\%) patients who did not have organ damage at baseline had damage accrual at the end of the study $(\mathrm{p}<0.01)$.

Univariate logistic regression analysis showed that high disease activity (AMS $>4$ ), baseline SDI and higher quartiles of cumulative prednisolone were each significantly associated with change in SDI (table 2). Multivariate analysis was performed to identify independent factors influencing damage accrual, and ORs were adjusted for ethnicity, AMS, baseline SDI and cumulative prednisolone quartiles. After adjustment, only a trend towards significance for the association of AMS $>4$ with damage accrual was observed (adjusted OR=1.88, 95\% CI $(0.81$ to 4.35), $\mathrm{p}=0.14$ ) (table 2). In contrast, baseline SDI remained strongly associated with damage accrual, with a threefold increase in risk of damage accrual (table 2). Cumulative prednisolone exposure was also strongly associated with damage accrual. Patients in the highest quartile of cumulative prednisolone had an OR for damage accrual of over 10 compared with those in the lowest quartile (adjusted OR=13.46, 95\% CI (3.59 to
50.4), $\quad \mathrm{p}<0.01)$. A dose-response relationship was observed between cumulative prednisolone use and damage accrual, with increasing ORs with each ascending quartile, and the third quartile of cumulative prednisolone use also statistically significant for damage accrual (table 2). When compared with Caucasians, Asian ethnicity was associated with reduced odds of damage accrual (adjusted OR=0.22, 95\% CI (0.09 to $0.53), \mathrm{p}<0.01$ ) (table 2). No statistically significant associations between damage accrual and other demographic factors including gender, age, and current smoking were found. As time-adjusted mean PGA was highly correlated with AMS, we did not include PGA in the multivariable analysis reported here; when included in the analysis, there was no effect on the associations of glucocorticoid use with damage accrual (data not shown).

Factors associated with damage accrual in glucocorticoid-related and non-glucocorticoid related domains

Fourteen patients developed damage in GC-SDI domains, including 12 patients exposed to

Table 2 Factors associated with damage accrual $(n=162)$

\begin{tabular}{|c|c|c|c|c|}
\hline & \multicolumn{2}{|c|}{$\begin{array}{l}\text { Unadjusted OR for damage } \\
\text { accrual }(\Delta S D \mid>0)\end{array}$} & \multicolumn{2}{|c|}{$\begin{array}{l}\text { Adjusted* OR for damage accrual } \\
(\Delta \mathrm{SDl}>0)\end{array}$} \\
\hline & OR (95\% Cl) & p Value & OR (95\% Cl) & p Value \\
\hline \multicolumn{5}{|l|}{ Gender } \\
\hline Female & 1 & & & \\
\hline Male & $1.38(0.57$ to 3.35$)$ & 0.5 & & \\
\hline \multicolumn{5}{|l|}{ Age at recruitment (years) } \\
\hline$<40$ & 1 & & & \\
\hline$\geq 40$ and $\leq 59$ & $0.96(0.47$ to 1.95$)$ & 0.9 & & \\
\hline$\geq 60$ & $1.29(0.51$ to 3.22$)$ & 0.6 & & \\
\hline \multicolumn{5}{|l|}{ Ethnicity } \\
\hline Caucasian & 1 & & & 1 \\
\hline Asian & $0.44(0.22$ to 0.89$)$ & 0.02 & $0.22(0.09$ to 0.53$)$ & $<0.01$ \\
\hline Other & $0.81(0.18$ to 3.58$)$ & 0.8 & $0.73(0.13$ to 4.24$)$ & 0.73 \\
\hline \multicolumn{5}{|l|}{ Baseline SDI } \\
\hline $\mathrm{SDI}=0$ & 1 & & & 1 \\
\hline $\mathrm{SDI} \geq 1$ & $3.12(1.58$ to 6.17$)$ & $<0.01$ & $3.10(1.40$ to 6.86$)$ & $<0.01$ \\
\hline \multicolumn{5}{|l|}{ Current smoker? } \\
\hline No & 1 & & & \\
\hline Yes & $1.33(0.56$ to 3.18$)$ & 0.5 & & \\
\hline \multicolumn{5}{|l|}{ Time-adjusted mean SLEDAI (AMS) } \\
\hline $\mathrm{AMS} \leq 4$ & 1 & & & 1 \\
\hline $\mathrm{AMS}>4$ & 3.18 (1.63 to 6.22$)$ & $<0.01$ & $1.88(0.81$ to 4.35$)$ & 0.14 \\
\hline \multicolumn{5}{|c|}{ Time-adjusted median PGA (TAM-PGA) } \\
\hline TAM-PGA $\leq 1$ & 1 & & & \\
\hline TAM-PGA>1 & $2.35(0.69$ to 8.08$)$ & 0.17 & & \\
\hline \multicolumn{5}{|l|}{ Cumulative prednisolone (mg) } \\
\hline First quartile $(0,0)$ & 1 & & & 1 \\
\hline Second quartile $(168,4568)$ & $1.94(0.63$ to 5.98$)$ & 0.2 & $2.19(0.65$ to 7.43$)$ & 0.21 \\
\hline Third quartile $(5054,9205)$ & 3.73 (1.28 to 10.9$)$ & 0.02 & 3.56 (1.07 to 11.8$)$ & 0.04 \\
\hline Fourth quartile $(9310,56635)$ & 9.72 (3.31 to 28.5$)$ & $<0.01$ & 13.46 (3.59 to 50.4$)$ & $<0.01$ \\
\hline
\end{tabular}


glucocorticoids during the study period (table 1). In multivariate analysis, higher cumulative prednisolone exposure was associated with GC-SDI accrual, with an OR of the highest quartile prednisolone exposure for GC-SDI accrual of over 9 (OR: 9.79 (95\% CI 1.43 to 66.8), $\mathrm{p}=0.02$ ) (table 3). Ethnicity, baseline SDI and AMS were not significantly associated with damage accrual in the GC-SDI (table 3).

We also analysed damage accrual in the residual domains of SDI after subtraction of GC-SDI, the 'non-GC-SDI'. In univariate analysis, non-GC-SDI accrual was associated with baseline SDI, AMS and with cumulative glucocorticoid dose, and negatively associated with Asian ethnicity (table 3). In multivariable analysis, non-GC-SDI remained significantly associated with baseline SDI (OR=3.09 (95\% CI 1.36 to 7.04), $\mathrm{p}<0.01), \quad \mathrm{AMS}>4 \quad(\mathrm{OR}=2.53 \quad(95 \%$ CI 1.07 to 6.02), $\mathrm{p}=0.04)$ and with cumulative prednisolone exposure, with an OR of 9.21 (95\% CI 2.39 to 35.5, $\mathrm{p}<0.01)$ for the highest compared with the lowest quartile of glucocorticoid exposure (table 3 ). The negative association of Asian ethnicity with non-GC-SDI accrual also remained significant after adjustment (table 3). Inclusion of PGA in the multivariate analysis did not impact on the significance of associations of glucocorticoid exposure with damage accrual in either domain (data not shown).

In supplementary analyses (see online supplementary tables S1 and S2), we substituted timeadjusted mean prednisolone for cumulative prednisolone exposure and found that associations of overall damage accrual with glucocorticoid use remained significant using this descriptor. In multivariable analysis controlling for baseline SDI, AMS and ethnicity, we confirmed an independent association of overall damage accrual with time-adjusted mean prednisolone that strengthened with higher mean daily dosage. Time-adjusted mean doses of prednisolone in the upper two quartiles of daily dose, that is, $>4.42 \mathrm{mg} /$ day or $>7.82 \mathrm{mg} /$ day, were associated with significantly increased overall damage accrual, and the highest risk of damage accrual in non-GC-SDI was observed in the highest quartile of glucocorticoid exposure, a timeadjusted mean daily dose $\geq 7.82 \mathrm{mg} /$ day. Cumulative glucocorticoid exposure was not analysed as a continuous variable given the skewed distribution of the data. We also analysed the frequency of damage accrual events in organ-specific subdomains of the SDI according to glucocorticoid exposure (see online supplementary table S3) and found no statistically significant trend in the association of glucocorticoid use with any individual organ domain.

\section{DISCUSSION}

Glucocorticoids are commonly prescribed in SLE because of their rapid and broad-spectrum actions on the immune system. ${ }^{18}$ Although they have predictable dose-dependent adverse effects, glucocorticoids continue to be widely used in SLE because of a lack of suitable alternatives. Given emerging evidence of harm associated with the use of glucocorticoids in SLE, ${ }^{7} 19$ reduction in glucocorticoid dose has been used as a secondary endpoint in clinical trials and recommended as part of a treat-to-target approach. ${ }^{20}{ }^{21}$ However, the nexus between the beneficial and harmful effects of glucocorticoids in SLE is incompletely understood. We undertook a study of predictors of damage accrual in a longitudinally followed cohort of patients with SLE, with a focus on glucocorticoid use. We report that glucocorticoids are associated with overall damage, glucocorticoidinduced damage and damage in domains of the SDI not traditionally associated with glucocorticoid-induced harm, independently of disease activity.

This study was undertaken in a longitudinal SLE cohort in which disease activity, damage and drug treatment data are collected prospectively. ${ }^{11}{ }^{12}$ The median period of observation was 4 years, and the median AMS was 3.5; this was sufficient to see the accrual of irreversible organ damage, as measured using change in SDI, in approximately one-third of patients. As expected, factors significantly associated with damage accrual in univariate analysis included baseline damage score and a timeadjusted measure of disease activity, AMS. In multivariate analysis, baseline SDI was significantly and independently predictive of damage accrual in overall and non-GC-SDI domains, but not GC-SDI. AMS only trended towards significance in the covariate-adjusted regression model for overall damage, but as expected was significantly associated with damage accrual measured using the non-GC-SDI.

The association of glucocorticoid use with damage accrual was analysed in several ways. Initially, damage accrual was compared in patients with and without glucocorticoid exposure. Glucocorticoid exposure was significantly associated with increased likelihood of damage accrual. Second, in a categorical analysis of cumulative glucocorticoid exposure, we found a strong relationship between higher exposure and damage accrual. Patients whose glucocorticoid exposure fell in either of the two highest quartiles had significantly increased damage accrual; the time-adjusted mean daily prednisolone dose at the interface of the second and third quartiles was only $4.42 \mathrm{mg} /$ day. This is a lower threshold for the association of glucocorticoids with damage accrual than that recently reported by Thamer et $a l^{10}{ }^{10}$ who studied a prespecified low dose cut-off of $6 \mathrm{mg} /$ day and found that doses below this were not associated with damage. We analysed our data using this cut-off, and unlike Thamer et al, ${ }^{10}$ we found this cut-off did not separate patients who did and did not accrue damage (data not shown). Indeed, our results indicate that the highest quartile of time-adjusted mean daily dose, associated with highest risk of damage accrual in both GC-SDI and non-GC-SDI domains, included patients with mean daily doses as low as $7.8 \mathrm{mg} /$ day. Our 
Table 3 Factors associated with GC-SDI and non-GC-SDI damage accrual

\begin{tabular}{|c|c|c|c|c|c|c|c|c|}
\hline & \multicolumn{4}{|l|}{ GC-SDI accrual } & \multicolumn{4}{|l|}{ Non-GC-SDI accrual } \\
\hline & \multicolumn{2}{|l|}{$\begin{array}{l}\text { Unadjusted OR for } \\
\Delta \text { GC-SDI>0 }\end{array}$} & \multicolumn{2}{|c|}{ Adjusted $^{*}$ OR for $\Delta \mathrm{GC}-\mathrm{SDI}>0$} & \multicolumn{2}{|l|}{$\begin{array}{l}\text { Unadjusted OR for } \\
\Delta \text { non-GC-SDI>0 }\end{array}$} & \multicolumn{2}{|l|}{$\begin{array}{l}\text { Adjusted† OR for } \\
\Delta \text { non-GC-SDI>0 }\end{array}$} \\
\hline & OR (95\% Cl) & p Value & OR $(95 \% \mathrm{Cl})$ & p Value & OR (95\% Cl) & p Value & OR (95\% Cl) & p Value \\
\hline \multicolumn{9}{|l|}{ Ethnicity } \\
\hline Caucasian & 1 & & 1 & & 1 & & 1 & \\
\hline Asian & $0.38(0.10$ to 1.45$)$ & 0.16 & $0.28(0.07$ to 1.16$)$ & 0.08 & $0.47(0.22$ to 0.97$)$ & 0.04 & $0.24(0.10$ to 0.60$)$ & $<0.01$ \\
\hline Other & $1.13(0.13$ to 10.2$)$ & 0.9 & $1.20(0.11$ to 12.9$)$ & 0.88 & $1.02(0.23$ to 4.54$)$ & 0.98 & 0.97 (0.17 to 5.64$)$ & 0.9 \\
\hline \multicolumn{9}{|l|}{ Baseline SDI } \\
\hline $\mathrm{SDI}=0$ & 1 & & 1 & & 1 & & 1 & \\
\hline $\mathrm{SDI} \geq 1$ & 2.64 (0.79 to 8.79$)$ & 0.11 & 2.90 (0.80 to 10.6$)$ & 0.11 & $3.23(1.58$ to 6.58$)$ & $<0.01$ & 3.09 (1.36 to 7.04$)$ & $<0.01$ \\
\hline \multicolumn{9}{|c|}{ Time-adjusted mean SLEDAI (AMS) } \\
\hline AMS $\leq 4$ & 1 & & 1.00 & & 1 & & 1 & \\
\hline AMS $>4$ & $1.35(0.45$ to 4.04$)$ & 0.6 & 0.58 (0.14 to 2.33$)$ & 0.44 & $3.93(1.94$ to 7.97$)$ & $<0.01$ & 2.53 (1.07 to 6.02$)$ & 0.04 \\
\hline \multicolumn{9}{|l|}{ Cumulative prednisolone $(\mathrm{mg})$} \\
\hline First quartile $(0,0)$ & 1 & & 1 & & 1 & & 1 & \\
\hline Second quartile $(168,4568)$ & $1.03(0.14$ to 7.66$)$ & 0.9 & $1.35(0.17$ to 10.5$)$ & 0.8 & $1.80(0.53$ to 6.06$)$ & 0.3 & $1.78(0.48$ to 6.60$)$ & 0.39 \\
\hline Third quartile $(5054,9205)$ & $1.00(0.13$ to 7.46$)$ & 0.9 & $1.27(0.16$ to 10.3$)$ & 0.8 & 4.15 (1.34 to 12.9$)$ & 0.01 & 3.39 (0.96 to 11.9$)$ & 0.06 \\
\hline \multirow[t]{4}{*}{ Fourth quartile $(9310,56635)$} & $4.87(0.97$ to 24.6$)$ & 0.06 & 9.79 (1.43 to 66.8$)$ & 0.02 & 8.80 (2.86 to 27.1$)$ & $<0.01$ & 9.21 (2.39 to 35.5$)$ & $<0.01$ \\
\hline & \multicolumn{4}{|c|}{ GC-SDI accrual $(n=14)$} & \multicolumn{4}{|c|}{ Non-GC-SDI accrual $(n=50)$} \\
\hline & \multicolumn{2}{|c|}{$\begin{array}{l}\text { Unadjusted OR for } \\
\Delta \text { GC-SDI>0 }\end{array}$} & \multicolumn{2}{|c|}{ Adjusted $^{*}$ OR for $\Delta \mathrm{GC}-\mathrm{SDl}>0$} & \multicolumn{2}{|c|}{$\begin{array}{l}\text { Unadjusted OR for } \\
\Delta \text { non-GC-SDI>0 }\end{array}$} & \multicolumn{2}{|l|}{$\begin{array}{l}\text { Adjusted† OR for } \\
\Delta \text { non-GC-SDI>0 }\end{array}$} \\
\hline & OR (95\% Cl) & p Value & OR (95\% Cl) & p Value & OR $(95 \% \mathrm{Cl})$ & p Value & OR (95\% Cl) & p Value \\
\hline \multicolumn{9}{|l|}{ Ethnicity } \\
\hline Caucasian & 1 & & 1 & & 1 & & 1 & \\
\hline Asian & $0.38(0.10$ to 1.45$)$ & 0.16 & $0.28(0.07$ to 1.16$)$ & 0.08 & $0.47(0.22$ to 0.97$)$ & 0.04 & 0.24 (0.10 to 0.60$)$ & $<0.01$ \\
\hline Other & 1.13 (0.13 to 10.2$)$ & 0.9 & $1.20(0.11$ to 12.9$)$ & 0.88 & $1.02(0.23$ to 4.54$)$ & 0.98 & 0.97 (0.17 to 5.64$)$ & 0.9 \\
\hline \multicolumn{9}{|l|}{ Baseline SDI } \\
\hline $\mathrm{SDI}=0$ & 1 & & 1 & & 1 & & 1 & \\
\hline $\mathrm{SDI} \geq 1$ & 2.64 (0.79 to 8.79$)$ & 0.11 & 2.90 (0.80 to 10.6$)$ & 0.11 & 3.23 (1.58 to 6.58$)$ & $<0.01$ & 3.09 (1.36 to 7.04$)$ & $<0.01$ \\
\hline \multicolumn{9}{|c|}{ Time-adjusted mean SLEDAI (AMS) } \\
\hline AMS $\leq 4$ & 1 & & 1.00 & & 1 & & 1 & \\
\hline AMS $>4$ & 1.35 (0.45 to 4.04$)$ & 0.6 & 0.58 (0.14 to 2.33$)$ & 0.44 & $3.93(1.94$ to 7.97$)$ & $<0.01$ & 2.53 (1.07 to 6.02$)$ & 0.04 \\
\hline \multicolumn{9}{|l|}{ Cumulative prednisolone $(\mathrm{mg})$} \\
\hline First quartile $(0,0)$ & 1 & & 1 & & 1 & & 1 & \\
\hline Second quartile $(168,4568)$ & $1.03(0.14$ to 7.66$)$ & 0.9 & $1.35(0.17$ to 10.5$)$ & 0.8 & $1.80(0.53$ to 6.06$)$ & 0.3 & $1.78(0.48$ to 6.60$)$ & 0.39 \\
\hline Third quartile $(5054,9205)$ & $1.00(0.13$ to 7.46$)$ & 0.9 & $1.27(0.16$ to 10.3$)$ & 0.8 & 4.15 (1.34 to 12.9$)$ & 0.01 & 3.39 (0.96 to 11.9$)$ & 0.06 \\
\hline Fourth quartile $(9310,56635)$ & 4.87 (0.97 to 24.6$)$ & 0.06 & 9.79 (1.43 to 66.8$)$ & 0.02 & 8.80 (2.86 to 27.1$)$ & $<0.01$ & 9.21 (2.39 to 35.5$)$ & $<0.01$ \\
\hline
\end{tabular}


findings suggest that only extremely low doses of glucocorticoid can be considered free of association with damage accrual in patients with SLE.

As expected, glucocorticoid-exposed patients had significantly worse disease activity, potentially confounding analysis of the association of glucocorticoid dose with damage. However, the association of cumulative glucocorticoid use with damage accrual remained significant after adjustment for disease activity and ethnicity as well as baseline SDI. An association of glucocorticoids with damage independent of disease activity has been documented in several recent studies. ${ }^{7} 8{ }^{10}{ }^{22-25}$ In 2014, Ruiz-Arruza reported prednisolone to be independently associated with damage in an ethnically homogeneous Spanish cohort. ${ }^{7}$ They did not report the contribution of disease activity to damage accrual, and disease activity was integrated into multivariate analysis using mean SLEDAI as opposed to the more informative AMS. A recent analysis of over 2000 patients with a mean observed period of 6.2 years in the Hopkins Cohort ${ }^{19}$ reported a $2.8 \%$ increased risk of developing new organ damage for each $1 \mathrm{mg}$ /day of prednisolone. An independent association of glucocorticoid use with damage accrual was also recently reported in the Systemic Lupus International Collaborating Clinics (SLICC) inception cohort, although in that study glucocorticoid use was only recorded as present or absent, ${ }^{24}$ preventing consideration of dose thresholds to damage accrual.

We also analysed a recently reported measure of glucocorticoid-related damage, derived from four putatively glucocorticoid-dependent domains of the SDI, known as the GC-SDI. ${ }^{7}$ The GC-SDI was first reported ${ }^{7}$ in a study which showed an adjusted OR that approached 10 for GC-SDI among patients with SLE exposed to prednisone $>7.5 \mathrm{mg} /$ day over 5 years. In our cohort, accrual of GC-SDI was twice as prevalent in patients that had received glucocorticoids compared with patients not exposed during the study period. Moreover, the OR for accrual of damage in the GC-SDI was $>12$ in patients in the highest quartile of cumulative glucocorticoid exposure, after adjusting for covariates including ethnicity, baseline SDI and disease activity; this equated to a time-adjusted mean daily dose of $7.82 \mathrm{mg} /$ day, remarkably similar to that reported by Ruiz-Arruza. Two patients who were not exposed to glucocorticoids during the study period accrued glucocorticoid-related damage; one patient who developed an osteoporotic fracture had been exposed to glucocorticoids prior to study entry, and the second developed cataracts.

Analysing for GC-SDI afforded us the opportunity to study the remaining domains of the SDI, which we notionally designated as the non-GC-SDI. Both univariate and multivariate analyses revealed a significant positive association between AMS and the accrual of damage in non-GC-SDI. Importantly, we also found that glucocorticoid use was highly significantly associated with damage accrual in this domain, and this association remained significant after adjusting for other variables. We conclude that glucocorticoid use is associated with damage accrual across the range of directly glucocorticoid-associated and disease-associated endpoints in SLE.

The possibility that glucocorticoid exposure exacerbates SLE organ damage in domains not traditionally associated directly with glucocorticoid use has not previously been suggested. There are several possible explanations for these observations. The first is that glucocorticoids fail to fully arrest the progress of damage caused by active SLE; however, the persistence of the association of damage with glucocorticoid exposure after adjusting for baseline SDI and SLEDAI-2K does not support this. An alternative explanation is that the SLEDAI-2K does not fully capture aspects of disease activity that place patients at risk of irreversible damage, and that glucocorticoid dose is a simply surrogate indicator of disease activity. The inclusion of PGA in the assessment of disease activity potentially overcomes the limitations of measures such as the SLEDAI-2K. Our analysis of associations of PGA with damage accrual, including its inclusion in the multivariable analysis model, did not support the conclusion that the contribution of glucocorticoids to damage accrual is simply a reflection of disease activity.

It is possible that glucocorticoid effects on the immune system include activating effects on cellular mechanisms that contribute to damage in SLE. Possible pathways include glucocorticoid-associated increases in macrophage migration inhibitory factor (MIF), expression of which is strongly associated with disease activity and damage in murine models of SLE and in the human disease; ${ }^{26-28}$ MIF, paradoxically, is induced by glucocorticoids and directly mediates cellular effects which oppose glucocorticoid inhibition of inflammation. ${ }^{29}{ }^{30}$ Studies of molecular pathways with potentially damaging effects in SLE that are either not suppressed by, or are indeed induced by, glucocorticoids might reveal novel therapeutic targets to prevent SLE organ damage.

We observed a significant association between Asian ethnicity and reduced damage accrual. The association of Asian ethnicity with increased disease activity has previously been reported in a study from this centre, ${ }^{11}$ but in that study, differences in SDI over time were not recorded. Studies from multiethnic SLE cohorts have not consistently replicated associations between damage accrual and Asian ethnicity. Two studies report increase in renal-related damage in Asian patients, ${ }^{31} 32$ whereas another study reported reduced damage accrual in Asians. ${ }^{33}$ The actual ethnic background of patients designated as Asian, and the method of designation, varies among these studies. However, the possibility that genetic or environmental factors associated with Asian ethnicity contribute to higher disease activity but protection from damage accrual in SLE is intriguing and requires further investigation.

Certain caveats apply to the interpretation of the current study. This was a single-centre study, although one undertaken in a cohort in which clinical data 
including glucocorticoid dose are recorded prospectively. Information on prednisolone use was gained through physician documentation of patients' self-report in the clinic. Unlike other medicines which can be measured in blood, adherence to glucocorticoids is difficult to measure. ${ }^{34}$ Glucocorticoid effects on body habitus may impact on adherence; low levels of adherence have been documented in one formal study. ${ }^{35}$ Inpatient intravenous methylprednisolone exposure in 11 patients was included in the analysis presented; exclusion of intravenous dosing did not alter the associations of glucocorticoid exposure with overall, GC-related or non-GC related damage accrual. The population that received intravenous methylprednisolone had significant differences to the population that did not (see online supplementary table S4), including higher disease activity, damage scores and overall glucocorticoid exposure; however, the low number of patients precluded analysis for specific impact of intravenous methylprednisolone exposure on damage beyond the contribution to overall exposure. The size of the study also prevented multivariable analysis of contributions of glucocorticoid exposure and disease activity to individual domains of the SDI, but it is possible that patients with low disease activity in the setting of high glucocorticoid dose, or vice versa, have particular patterns of damage accrual that our analysis did not detect. It is noted that the ability of SLEDAI-2K and PGA to capture all aspects of disease activity in SLE is imperfect, and therefore statistical adjustment for disease activity using these measures may not capture all the effects of disease on damage accrual. Further studies to determine the nature of these 'missing' contributors are required. Finally, although accrual of damage measured using the SDI has been shown to be clinically significant, ${ }^{3}$ the SDI assigns similar scores to clinically diverse phenomena, and the clinical impact of individual changes in domains of the SDI and their link to glucocorticoids are incompletely understood.

In conclusion, this study demonstrates that glucocorticoid use is strongly associated with the accrual of organ damage in SLE. The association of glucocorticoid exposure with damage accrual was independent of measures of disease activity and baseline damage, even when applied following the exclusion of the glucocorticoid-induced domains of the SDI. Results were most significantly associated above a time-adjusted mean dose $7.82 \mathrm{mg} /$ day, however, strikingly, were still significant above a very low dose of $4.42 \mathrm{mg} /$ day. Given the limitations of observational studies in the face of confounding by indication, our findings suggest the urgent need for a randomised study comparing the effect on damage accrual of usual care with that of a strategy that stringently limits glucocorticoid dosing. Our findings further emphasise the need for new, more effective treatments for SLE that minimise or eliminate the need for glucocorticoids.

Contributors All contributors meet the criteria for authorship and approved the submitted version of the manuscript.
Funding The work reported on this manuscript was supported in part by an unrestricted educational grant from Eli Lilly.

Competing interests None declared.

Patient consent Obtained.

Ethics approval Monash Health HREC Ref: 15212L.

Provenance and peer review Not commissioned; externally peer reviewed.

Data sharing statement No additional data are available.

Open Access This is an Open Access article distributed in accordance with the Creative Commons Attribution Non Commercial (CC BY-NC 4.0) license, which permits others to distribute, remix, adapt, build upon this work noncommercially, and license their derivative works on different terms, provided the original work is properly cited and the use is non-commercial. See: http:// creativecommons.org/licenses/by-nc/4.0/

\section{REFERENCES}

1. Rivest C, Lew RA, Welsing PM, et al. Association between clinical factors, socioeconomic status, and organ damage in recent onset systemic lupus erythematosus. J Rheumatol 2000;27:680-4.

2. Gladman D, Ginzler E, Goldsmith C, et al. The development and initial validation of the Systemic Lupus International Collaborating Clinics/American College of Rheumatology damage index for systemic lupus erythematosus. Arthritis Rheum 1996;39:363-9.

3. Chambers SA, Allen E, Rahman A, et al. Damage and mortality in a group of British patients with systemic lupus erythematosus followed up for over 10 years. Rheumatology (Oxford) 2009;48:673-5.

4. Mok CC, Ho CTK, Wong RWS, et al. Damage accrual in southern Chinese patients with systemic lupus erythematosus. J Rheumatol 2003;30:1513-19.

5. Urowitz MB, Gladman DD, Tom BDM, et al. Changing patterns in mortality and disease outcomes for patients with systemic lupus erythematosus. J Rheumatol 2008:2152-8.

6. Ibañez D, Gladman DD, Urowitz MB. Adjusted mean systemic lupus erythematosus disease activity Index-2K is a predictor of outcome in SLE. J Rheumatol 2005;32:824-7.

7. Ruiz-Arruza I, Ugarte A, Cabezas-Rodriguez I, et al. Glucocorticoids and irreversible damage in patients with systemic lupus erythematosus. Rheumatology 2014;53:1470-6.

8. Zonana Nacach A, Barr SG, Magder LS, et al. Damage in systemic lupus erythematosus and its association with corticosteroids. Arthritis Rheum 2000;43:1801-8.

9. Saag KG, Koehnke R, Caldwell JR, et al. Low dose long-term corticosteroid therapy in rheumatoid arthritis: an analysis of serious adverse events. Am J Med 1994;96:115-23.

10. Thamer M, Hernan MA, Zhang $Y$, et al. Prednisone, lupus activity, and permanent organ damage. J Rheumatol 2009;36:560-4.

11. Golder V, Connelly K, Staples M, et al. Association of Asian ethnicity with disease activity in SLE: an observational study from the Monash Lupus Clinic. Lupus 2013;22:1425-30.

12. Vincent FB, Northcott M, Hoi A, et al. Association of serum BAFF and APRIL with central nervous system and renal disease in systemic lupus erythematosus. Lupus 2013;22:873-84.

13. Hochberg MC. Updating the American college of rheumatology revised criteria for the classification of systemic lupus erythematosus. Arthritis Care Res (Hoboken) 1997;40:1725-5

14. Gladman DD, Ibañez D, Urowitz MB. Systemic lupus erythematosus disease activity index 2000. J Rheumatol 2002;29:288-91.

15. Ibanez D, Urowitz MB, Gladman DD. Summarizing disease features over time: I. Adjusted mean SLEDAI derivation and application to an index of disease activity in lupus. J Rheumatol 2003;30:1977-82.

16. Yee C-S, Farewell VT, Isenberg DA, et al. The use of Systemic Lupus Erythematosus Disease Activity Index-2000 to define active disease and minimal clinically meaningful change based on data from a large cohort of systemic lupus erythematosus patients. Rheumatology 2011:982-8.

17. Petri M, Kim MY, Kalunian KC, et al. Combined oral contraceptives in women with systemic lupus erythematosus. $N$ Engl J Med 2005;353:2550-8.

18. Fan H, Morand EF. Targeting the side effects of steroid therapy in autoimmune diseases: the role of GILZ. Discov Med 2012;13:123-33.

19. Al Sawah S, Zhang X, Zhu B, et al. Effect of corticosteroid use by dose on the risk of developing organ damage over time in systemic lupus erythematosus. Lupus Sci Med 2015;2:e000066. 
20. Franklyn K, Hoi A, Nikpour M, et al. The need to define treatment goals for systemic lupus erythematosus. Nat Rev Rheumatol 2014;10:567-71.

21. van Vollenhoven RF, Mosca M, Bertsias G, et al. Treat-to-target in systemic lupus erythematosus: recommendations from an international task force. Ann Rheum Dis 2014;73: 958-67.

22. Lopez R, Davidson JE, Beeby MD, et al. Lupus disease activity and the risk of subsequent organ damage and mortality in a large lupus cohort. Rheumatology (Oxford) 2012;51: 491-8.

23. Petri M, Purvey S, Fang $\mathrm{H}$, et al. Predictors of organ damage in systemic lupus erythematosus: the Hopkins Lupus Cohort. Arthritis Rheum 2012;64:4021-8.

24. Bruce IN, O'Keeffe AG, Farewell V, et al. Factors associated with damage accrual in patients with systemic lupus erythematosus: results from the Systemic Lupus International Collaborating Clinics (SLICC) Inception Cohort. Ann Rheum Dis 2015;74:1706-13.

25. Yee CS, Su L, Toescu V, et al. Birmingham SLE cohort: outcomes of a large inception cohort followed for up to 21 years. Rheumatology (Oxford) 2015;54:836-43. http://dx.doi.org/10.1093/ rheumatology/keu412

26. Foote A, Briganti EM, Kipen Y, et al. Macrophage migration inhibitory factor in systemic lupus erythematosus. J Rheumatol 2004;31:268-73.

27. Hoi AY, Hickey MJ, Hall $P$, et al. Macrophage migration inhibitory factor deficiency attenuates macrophage recruitment, glomerulonephritis, and lethality in MRL/Ipr mice. J Immunol 2006;177:5687-96.

28. Sanchez E, Gomez LM, Lopez-Nevot MA, et al. Evidence of association of macrophage migration inhibitory factor gene polymorphisms with systemic lupus erythematosus. Genes Immun 2006;7:433-6.

29. Fan $\mathrm{H}$, Kao W, Yang $\mathrm{YH}$, et al. Macrophage migration inhibitory factor inhibits the antiinflammatory effects of glucocorticoids via glucocorticoid-induced leucine zipper. Arthritis Rheum 2014;66:2059-70.

30. Aeberli D, Leech M, Morand EF. Macrophage migration inhibitory factor and glucocorticoid sensitivity. Rheumatology (Oxford) 2006;45:937-43.

31. Johnson SR, Urowitz MB, Ibañez D, et al. Ethnic variation in disease patterns and health outcomes in systemic lupus erythematosus. J Rheumatol 2006;33:1990-5.

32. Stoll T, Sutcliffe N, Klaghofer R, et al. Do present damage and health perception in patients with systemic lupus erythematosus predict extent of future damage?: a prospective study. Ann Rheum Dis 2000;59:832-5.

33. Peschken CA, Katz SJ, Silverman E, et al. The 1000 Canadian faces of lupus: determinants of disease outcome in a large multiethnic cohort. J Rheumatol 2009;36:1200-8.

34. Croyle L, Morand EF. Optimizing the use of existing therapies in lupus. Int $J$ Rheum Dis 2015;18:129-37.

35. Koneru S, Shishov M, Ware A, et al. Effectively measuring adherence to medications for systemic lupus erythematosus in a clinical setting. Arthritis Rheum 2007;57:1000-6. 